accounted for by the mental disease would have attracted any notice had not the thermometer been employed daily.

'The patient's appetite was good almost to the end, and she could take food on the last day. 'The bowels were inclined to looseness, but not too much so. She had a slight, hacking cough, but breathed until the last two weeks without difficulty.

The elevated temperature was the only indication that at first alarmed me. The mental disease could easily explain this for the first three weeks. After this, however, the continued high temperature and its extraordinary mobility between night and morning made me feel that there was a hidden complication. The physical signs pointed toward the end to phthisis, and the temperature was also indicative of this complication. From the fortieth to the forty-fifth day there were two hæmorrhages, one considerable one at night. Considerable pus was coughed up on several occasions. There was no autopsy.

This case demonstrated to a remarkable degree the utility of the thermometer in insanity, if complications exist. 'The patient's skin did not appear very warm and entirely deceived me as to its heat. The cheeks were often flushed at night, a hectic flush setting in pretty regularly about three o'clock, but the skin never suggested a higher temperature than 100 to 102 degrees. I always took this patient's temperature in the rectum, but sometimes doubting the correctness of the observations, would try other thermometers in the mouth and axilla, but with a similar result. The axillary temperature was a degree to a degree and one-half less than in the rectum.

The " amphibolic" stage of Wünderlich 7 was well marked in this case, beginning apparently about the twenty-seventh day. There was, at the end of this stage, a characteristic final rise of temperature or " perturbatio critica." The great irregularities in temperature were unfavorable from the start. Thus the temperature, by itself, indicated pretty surely a fatal result.

Clouston ${ }^{8}$ speaks of the temperature of phthisical insanity being higher than any other form of insanity. This would apply also to a case in which phthisis was developed in the course of an attack of insanity.

( To be concluded.)

\section{A CASE OF TUBERCULAR MENINGITIS: DEATH: AUTOPSY.1}

BY HENRY P. JAQUES, M.D., OF MILTON.

The patient was a boy, five years old. He was always well and strong, except for a tonsillitis, of which he had frequent attacks. Family history good on both sides.

On December 29, 1884, I was called to see him, and obtained the following history: He had come out to Milton looking and feeling as well as ever. On December 23d, while endeavoring to climb into bed, he fell and struck the back of his head. He

7 Seguin's Wünder]lch.

8 Oloservations on the temperature of the insane.

1 Read, by invitation, before the Obstetrical Socfety of Boston, March 14, 1885 . cried afterward, but the blow left no mark, and no more was thought of it. The next day, while playing, he fell and struck his forehead, but the blow was no more serious than he had often had before. That evening, Christmas eve, he went to a children's party, but ate nothing unusual, went to bed early, and slept all night. Seeing, and playing with, so many children was unusual for him, but he did not seem excited by it. For a day or two afterward he was constipated and somewhat fretful, both of which conditions were remarkable for him.

On December 29 th, the date of my first visit, he had a slight follicular tonsillitis. His pulse and temperature were normal ; cheeks flushed; eyes dull ; pupils normal. His head was slightly hot, and he was dull and drowsy. He had had no dejection for two days. I prescribed for his throat and his bowels, and told the family that he was not very sick, though I did not like the drowsiness, which, they said, was unusual. The next day his bowels moved, and I heard no more from him for several days, and thought they had probably taken him back to Boston. On January $2 d$ they sent for their family physician, Dr. W. L. Richardson. He found the child still drowsy and again constipated, but no other symptoms. His throat was not nearly as bad as in his former attacks. He ordered cathartics, to be followed, if necessary, by injections. 'That evening I was again sent for. I found that the constipation was not relieved, and that the drowsiness had increased to such an alarming extent that he had to be roused before answering questions. His head was hot, and he complained of some pain in the back of his head. His face was flushed, and the eyes vacant and staring. Pulse 64 and strong, and the temperature $99^{\circ} \mathrm{F}$. The bowels were moved by injections, and he passed water, though with difficulty. I told the family that there was something more than mere constipation, and advised them to send for Dr. Richardson early in the morning. During the night he slept only at intervals. Vomited once. One dejection, coming away with considerable flatus. Passed water twice freely. He moved his left leg spasmodically, and clenched his hands occasionally. He was uneasy, and moaned. Head hot. Pulse at times intermittent. Gradually he became more and more drowsy, and finally relapsed into a state of unconsciousness.

In the morning I saw him with Dr. Richardson. A careful examination revealed nothing abnormal in the chest or abdomen. The child was completely unconscious. Pupils normal. Temperature $100.6^{\circ}$; pulse 108 ; respiration 30 .

We told the family that the case was very serious, that it was probably some form of meningitis, and advised them to send for Dr. T. M. Rotch. An icebag was applied to the head, and enemata of one ounce of milk and one drachm of brandy ordered every two hours.

In the evening Dr. Rotch, on examination, found no new symptoms except a slight congestion in the right ear in the neighborhood of the malleus. On the back, over the right apex, there was a slight elevation of pitch on percussion, but no other physical signs. Temperature $101.2^{\circ}$; pulse 89 , and strong.

Dr. Rotch agreed that the only diagnosis possible at this time was a probable meningitis, though it 
was possible that the right apex might develop new signs on further examination, and the symptoms be due to a hidden pneumonia.

During the day there was considerable twitching of the arms and legs, chiefly the right side, lasting from ten to twenty minutes, the chest being slightly raised. The pupils were slightly contracted and alike.

During the night he drew his right hand across the face with a quick, trembling motion, the right leg being drawn up and the whole body trembling. Occasional moaning and sighing respiration. Temperature, pulse, and respiration not much above normal. When the lips were bathed he partly opened his eyes and made an effort to swallow. Pupils sometimes contracted, sometimes dilated, cheeks flushed, tongue and lips dry. All enemata retained.

Dr. C. J. Blake, having been asked to examine the condition of the ears, found a slight congestion in the posterior canal of both ears, and also in the neighborhood of the right malleus. Dr. Blake thought that the congestion was merely a symptom of the meningeal congestion, and not the cause of the disease. When the speculum was inserted the patient showed signs of consciousness, slightly moving the eyebrows and facial muscles.

On January 6th Dr. S. G. Webber was asked to see the child in consultation. After a careful examination he decided that it was undoubtedly a case of meningitis. Taking into consideration the history of the falls and the blows on the head, and the excitement of the children's party afterward, he thought it probably a simple acute meningitis, though there was a possibility of its being tubercular. He recommended the trial of the iodide of potash. The congestion in posterior canals of both ears had diminished considerably. Pain was evidently caused by the examination.

During the next few days the condition varied but little. 'The enemata were almost all retained. The bowels moved once or twice daily, and the urine was passed regularly. The eyes, usually closed, were occasionally opened, with the eyeballs moving from side to side. The respiration sighing, interrupted, occasionally almost inaudible, then for a time noisy. At times the breathing was suspended for several seconds, bright red spots appearing on the cheeks, which disappeared when respiration was resumed. Patient moaned occasionally, and there was some moving and twitching of the extremities, though no convulsion. Pulse fair, though at times intermittent. The temperature, pulse, and respiration are all noted on the accompanying chart, so I will not recount here the daily variations. 'The extremities of the right side were absolutely motionless, and did not respond to the usual tests.

January 9 th. The extremities became cold, the face very pale, and the pulse imperceptible. This condition lasted fifteen minutes, when he improved in appearance. During the night the breathing grew very rapid, he was restless, moved the left arm continually, and moaned. After some time he opened his eyes, looked round the room, and then became quiet and slept. The next day he was more unconscious, and the fingers were flexed with a very strong contraction of the muscles. Breathing was more difficult, with the nostrils widely dilated at every breath. During the night he seemed fully conscious for some time, swallowed water without difficulty, and the eyes wide open.

January 11th. Ptosis of right eyelid. The pulse irregular, compressible, and intermittent. The left arm was occasionally raised to the head with a quick, spasmodic motion, the child moaning as if distressed meanwhile. Later, his eyes were fixed, pupils dilated, nostrils expanded, and bluish color around nose and mouth. The breathing was very difficult. During an attack of this kind he had every appearance of being moribund, and each attack was thought to be his last. One of the most remarkable features of the rase was the fact that the change from day to day was almost imperceptible, and to one who did not see the child it is almost impossible to give an adequate idea of his condition.

January 12th. The pupils of both eyes were much dilated. The right eye was almost motionless, with ptosis of the right lid, while the left eye moved rapidly from side to side in a circle. The face was livid, with the hands mottled with bright red spots. Later, the left eye became quiet, with a slightly contracted pupil. The next day the movements of the left eye were repeated, the right pupil being dilated and the left contracted. During all this time the enemata were retained, the bowels moved, and the urine was passed. The pulse was so weak that at times it could not be felt at all at the wrist, and the breathing was at times inaudible and almost imperceptible. Respiration sighing. Dr. Richardson and $I$ agreed that it was a case of probable tubercular meningitis, and held out no hope whatever to the family.

January 17th. There was a slight discharge of pus from the mouth, and also from the left eye. During the next day his breathing grew more and more difficult, and it seemed as if he could not possibly last much longer. In the evening, however, his respiration was much easier, and his whole appearance improved. His breath was very offensive, and there was a loud bubbling sound in the throat.

January 19th. The right nostril was much more dilated during inspiration than the left. The forehead was shiny, slightly odematous, and the veins plainly mapped out. Occasionally he moved his right hip-joint and shoulder, which had been motionless for days. There was another slight discharge of pus from the mouth, and when his lips were wiped he seemed more sensitive to touch than before. During the night his left arm and leg were constantly moved, and he moaned as if in pain. Forehead still cedematous. During the next night he was in a state of deep coma for four hours, then drew a deep sigh and seemed more conscious afterward. Pulse soft, intermittent, and fluttering.

January 20th. He partly opened and shut his right eye, which was very sensitive to light. Breathing was difficult and noisy, and the face covered with perspiration. At 10 P.M. sighing respiration began again, and at 10.15 he quietly died.

The case is interesting for the following reasons : The family history is good. The child's previous 
health was perfect, except for the frequent attacks of tonsillitis.

'The diagnosis was complicated, at first, by the doubt as to the part played in the causation of the disease by the blows on the head and the subsequent excitement. The duration of the state of unconsciousness - twenty days - was remarkable, though not unprecedented. Lastly, the enemata were almost all retained, the bowels moved, and the urine passed regularly, throughout the disease.

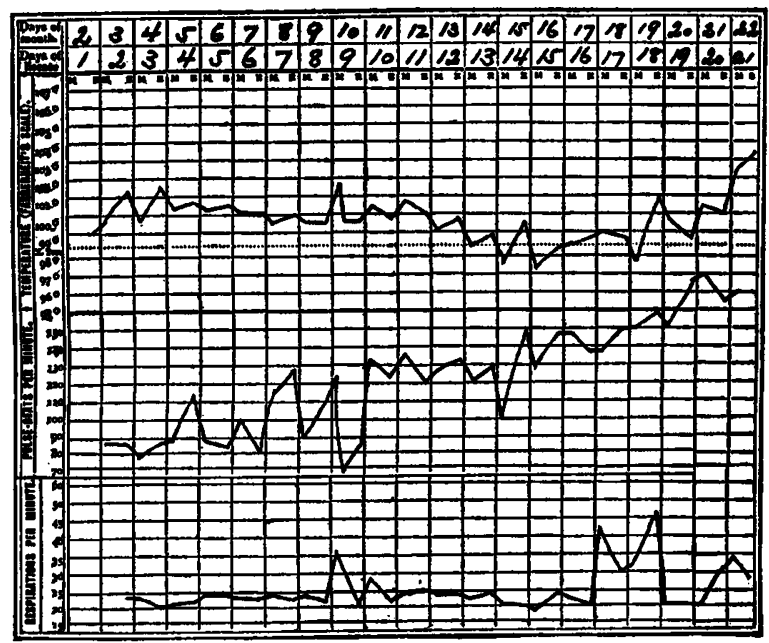

'The temperature was taken by the rectum, but, on comparison, was found to vary but slightly from that taken in the axilla.

The autopsy was made, eighteen hours after death, by Dr. W. W. Gannett.

Body medium sized (for age); well developed; much emaciated; slight lividity of dependent portions ; rigor mortis present.

Nothing unusual observed about the calvaria or dura; the sinuses of the latter contained partly coagulated blood.

The pia of the convexities of the brain very dry; the vessels minutely injected. The convolutions flattened, the sulci obliterated. The pia of the base along the chiasma and in the fissures of Sylvius was thickened, and opaque yellowish-gray from the presence of fibrino-purulent material in its meshes. In the above situations, also on the under surface of the frontal and temporal lobes, also on the pons and inner borders of the occipital lobes, were to be seen very numerous gray, translucent nodules the size of a pin's head. The lateral ventricles contained each about fifty cc. of a slightly opaque fluid. The ependyma thickened, grayish, and opaque. The choroid plexuses and velum interpositum markedly injected. In the latter were to be seen several small nodules similar to those described in connection with the pia of the base.

Section of the hemispheres showed nothing remarkable, the puncta cruenta being of about the usual size and number.

The basal ganglia, pons, medulla, and cerebellum also showed no appearances worthy of special note.

The heart of the usual size, and so far as its valves, cavities, and muscular substance were concerned showed nothing unusual.
'The pleural surfaces on both sides free from adhesions ; the pleural cavities contained no fluid.

Both lungs retracted; everywhere crepitant except at apices, where small nodules could be felt within the lung.

On section, at the top of the left lung, was an opaque, grayish-yellow, cheesy nodule, the size of a pea, surrounded by a narrow border of gray and translucent tissue. At the top of the right lung were several closely aggregated nodules of a similar appearance, forming, together, a mass the size of a small walnut. Otherwise the lungs showed nothing remarkable.

The bronchial lymph-glands were enlarged to the size of filberts, showing, on section, a yellowish, opaque, crumbling material.

The spleen was of the usual size, color, and density. On section, follicles and trabeculæ fairly distinct, pulp firm, pale-red in color. Two or three gray, translucent, sharply defined, slightly projecting nodules, the size of the prick of a pin were to be seen.

Kidneys not remarkable.

In the lower third of the ileum were several losses of substance of the mucous membrane; the edges elevated, the bases granular.

Liver showed nothing unusual.

Diagnosis. - Tubercular meningitis; acute hydrocephalus ; ependymitis ; tuberculosis of velum interpositum ; tubercular nodules in the lungs ; tuberculosis of bronchial lymph-glands; tuberculosis of the spleen; tubercular ulceration of the intestines.

\section{REPOR'T ON SURGERY.}

$$
\text { BY А. T. САBOT, А.M., M.D. }
$$

GUNSHOT WOUNDS OF THE ABDOMEN.

Considerable experience has been gained in the past six months in the matter of the treatment of these injuries.

Dr. William ' $T$. Bull reports 1 " a case of gunshot wound of the intestines treated successfully by laparotony with suture of the intestines."

'The patient, a man of twenty-two, received a pistol-shot wound entering the abdomen below and a little to the left of the umbilicus.

There was no immediate collapse. 'Twelve hours after the injury his pulse was 102 , his temperature was $100.2^{\circ} \mathrm{F}$. , and his respiration 30 . At this time he had severe abdominal pain in spite of having received about three fourths of a grain of morphia subcutaneously. The abdomen was normal in appearance, and palpation revealed nothing except the existence of universal tenderness. He vomited a little watery fluid and the urine which was drawn contained no blood.

Five hours later (seventeen hours after the wound) his condition was still the same, and Dr. Bull performed laparotomy under strict antiseptic precautions.

When the peritonæum was opened, at least two pints of bloody serum containing little clots but no fœcal matter flowed out.

The intestines were congested and were here and 1 Philadelphia Medical News, February 14, 1885. 